\title{
Three-dimensional orbits of the triple-O stellar system HD $150136^{\star}$
}

\author{
H. Sana ${ }^{1}$, J.-B. Le Bouquin ${ }^{2}$, L. Mahy ${ }^{3}$, O. Absil ${ }^{3}$, M. De Becker ${ }^{3}$, and E. Gosset ${ }^{3, \star \star}$ \\ 1 Astronomical Institute Anton Pannekoek, Amsterdam University, Science Park 904, 1098 XH, Amsterdam, The Netherlands \\ e-mail: h.sana@uva.nl \\ 2 UJF-Grenoble 1/CNRS-INSU, Institut de Planétologie et d'Astrophysique de Grenoble (IPAG) UMR 5274, Grenoble, France \\ 3 Département d'Astrophysique, Géophysique et Océanographie, Université de Liège, Allée du 6 Août 17, 4000 Liège, Belgium
}

Received 30 January 2013 / Accepted 3 April 2013

\begin{abstract}
Context. HD 150136 is a triple hierarchical system and a non-thermal radio emitter. It is formed by an O3-3.5 V + O5.5-6 V close binary and a more distant $06.5-7 \mathrm{~V}$ tertiary. So far, only the inner orbital properties have been reliably constrained.

Aims. To quantitatively understand the non-thermal emission process, accurate knowledge of the physical and orbital properties of the object is crucial. Here, we aim to investigate the orbital properties of the wide system and to constrain the inclinations of the inner and outer binaries, and with these the absolute masses of the system components.

Methods. We used the PIONIER combiner at the Very Large Telescope Interferometer to obtain the very first interferometric measurements of HD 150136. We combined the interferometric observations with new and existing high-resolution spectroscopic data to derive the orbital solution of the outer companion in the three-dimensional space.

Results. The wide system is clearly resolved by PIONIER, with a projected separation on the plane of the sky of about 9 milli-arcsec. The best-fit orbital period, eccentricity, and inclination are $8.2 \mathrm{yr}, 0.73$, and $108^{\circ}$. We constrain the masses of the three stars of the system to $63 \pm 10,40 \pm 6$, and $33 \pm 12 M_{\odot}$ for the O3-3.5 V, O5.5-6 V, and O6.5-7 V components.

Conclusions. The dynamical masses agree within errors with the evolutionary masses of the components. Future interferometric and spectroscopic monitoring of HD 150136 should allow one to reduce the uncertainties to a few per cent only and to accurately constrain the distance to the system. This makes HD 150136 an ideal system to quantitatively test evolutionary models of high-mass stars as well as the physics of non-thermal processes occurring in O-type systems.
\end{abstract}

Key words. binaries: close - binaries: spectroscopic - radiation mechanisms: non-thermal - stars: early-type stars: fundamental parameters - stars: massive

\section{Introduction}

HD 150136 is the brightest member of the NGC 6193 cluster in the Ara OB1 association and a known non-thermal radio-emitter (Benaglia et al. 2006), i.e. an object where particles are accelerated to relativistic energies (see De Becker 2007, and references therein). This distinctive feature was the reason for a decadelong observational effort to clarify the nature and properties of HD 150136. Recently, Mahy et al. (2012, hereafter Paper I) showed that HD 150136 is a triple hierarchical system consisting of an inner binary, with an $\mathrm{O} 3 \mathrm{~V}\left(\left(\mathrm{f}^{\star}\right)\right)-\mathrm{O} 3.5 \mathrm{~V}\left(\left(\mathrm{f}^{+}\right)\right)$primary star and an 05.5-6V((f)) secondary star on a $P_{\text {in }} \approx 2.67 \mathrm{~d}$ orbit, and a third physically bound $\mathrm{O} 6.5-7 \mathrm{~V}((\mathrm{f}))$ companion on a $P_{\text {out }} \approx 8$ - to 15 -year orbit. With a total mass estimated to be around $130 M_{\odot}$, HD 150136 is one of the most massive multiple O-star systems known. It is also the closest one to Earth to harbour an $\mathrm{O} 3$ star.

Given the range of possible orbital periods for the outer system, Mahy et al. (2012) estimated a probable separation on the plane of the sky between the inner pair and the third component of roughly 10 to 40 milli-arcsec (mas). Here, we report on extended spectroscopic monitoring that allows us to obtain the first orbital solution of the outer system. We also report on the very

$\star$ Based on observations collected at the European Southern Observatory (ESO) under program IDs 089.D-0730 and 189.C-0644.

$\star \star$ Senior Research Associate F.R.S.-FNRS. first interferometric detection of the wide system using the Very Large Telescope Interferometer (VLTI).

\section{Observations and data reduction}

\subsection{Spectroscopy}

We used the FEROS spectrograph mounted at the MPG/ESO $2.2 \mathrm{~m}$ telescope at La Silla (Chile) to obtain new high-resolution optical spectra of HD 150136 that supplement the FEROS data analysed in Paper I. Eighteen FEROS spectra were obtained during a eight-night run in May 2012 (PI: Mahy), providing a continuous wavelength coverage from 3700 to $9200 \AA$ at a resolving power of 48000 . The data were processed as described in Paper I. In addition, we searched the ESO archives for complementary data. We retrieved six FEROS spectra from 2011 (PI: Barbá; 087.D-0946(A)), one FEROS spectrum from 2008 (PI: Barbá; 079.D-0564(B)) and one UVES spectrum from July 2000 (PI: Roueff; 065.I-0526(A)). The latter two spectra provide additional constraints on the tertiary component, but not on the systemic velocity of the inner pair and were therefore used only to calculate the orbital solution of the wider pair.

The disentangling procedure described in Paper I for triple systems was applied to the 2011 and 2012 sets of spectra. We also reprocessed the complete data set, using our crosscorrelation technique on all data from 1999 to 2012 to consistently measure the radial velocities (RVs) of the HD 150136 
Table 1. Journal of the new and archival spectroscopic observations.

\begin{tabular}{|c|c|c|c|}
\hline $\begin{array}{l}\text { HJD } \\
-2450000\end{array}$ & $\begin{array}{c}v_{1} \\
\left(\mathrm{~km} \mathrm{~s}^{-1}\right)\end{array}$ & $\begin{array}{c}v_{2} \\
\left(\mathrm{~km} \mathrm{~s}^{-1}\right)\end{array}$ & $\begin{array}{c}v_{3} \\
\left(\mathrm{~km} \mathrm{~s}^{-1}\right)\end{array}$ \\
\hline \multicolumn{4}{|c|}{2000 UVES observation } \\
\hline 1726.5548 & 173.5 & -368.8 & 29.4 \\
\hline \multicolumn{4}{|c|}{2008 FEROS observation } \\
\hline 4658.5184 & -107.5 & 128.2 & 27.0 \\
\hline \multicolumn{4}{|c|}{2011 FEROS observations } \\
\hline $\begin{array}{l}5642.916 \\
5696.790 \\
5696.907 \\
5697.897 \\
5699.583 \\
5699.588\end{array}$ & $\begin{array}{r}-182.9 \\
-208.8 \\
-191.8 \\
186.5 \\
-198.9 \\
-191.7\end{array}$ & $\begin{array}{r}268.4 \\
298.5 \\
269.4 \\
-337.0 \\
272.9 \\
268.9\end{array}$ & $\begin{array}{l}-6.9 \\
-4.0 \\
-7.7 \\
-6.4 \\
-6.4 \\
-6.7\end{array}$ \\
\hline \multicolumn{4}{|c|}{2012 FEROS observations } \\
\hline 6048.661 & 148.6 & -274.7 & -23.7 \\
\hline 6048.758 & 120.7 & -221.2 & -27.4 \\
\hline 6048.899 & 62.0 & -150.9 & -29.5 \\
\hline 6049.597 & -215.8 & 306.1 & -18.0 \\
\hline 6049.715 & -221.1 & 314.8 & -18.0 \\
\hline 6049.924 & -208.2 & 280.1 & -22.3 \\
\hline 6050.666 & 119.1 & -223.4 & -26.2 \\
\hline 6050.843 & 167.7 & -310.1 & -25.7 \\
\hline 6051.641 & 33.6 & -114.2 & -22.1 \\
\hline 6051.864 & -107.5 & 128.2 & -21.0 \\
\hline 6052.646 & -192.7 & 268.9 & -20.4 \\
\hline 6052.781 & -152.4 & 219.6 & -24.9 \\
\hline 6052.909 & -109.9 & 107.7 & -27.6 \\
\hline 6053.660 & 188.6 & -344.1 & -27.6 \\
\hline 6053.778 & 187.8 & -350.7 & -28.3 \\
\hline 6053.937 & 168.4 & -308.4 & -26.1 \\
\hline 6054.649 & -152.4 & 190.2 & -26.5 \\
\hline 6054.758 & -188.9 & 240.8 & -23.4 \\
\hline
\end{tabular}

components. The RV values corresponding to the new observational epochs are given in Table 1 along with the journal of the 2011 and 2012 observations.

\subsection{Long baseline interferometry}

Interferometric data were obtained in June and August 2012 with the PIONIER combiner (Le Bouquin et al. 2011) and the Auxiliary Telescopes (ATs) at the VLTI (Haguenauer et al. 2010). Fringes were dispersed over three spectral channels across the $H$-band $(1.50-1.80 \mu \mathrm{m})$. The ATs were located in configuration A1-G1-K0-I1, providing six projected baselines ranging from 30 to $120 \mathrm{~m}$ and a maximum angular resolution of 2 mas in the $H$-band. Data were reduced and calibrated with the pndrs package (Le Bouquin et al. 2011). Calibration stars were chosen in the JMMC Stellar Diameters Catalog (Lafrasse et al. 2010). The closure phases and visibilities were modelled with two unresolved sources because the expected diameters of the individual components $(<0.1$ mas $)$ as well as the separation of the inner pair $(<0.2$ mas $)$ are largely unresolved by the longest VLTI baselines. The data show no evidence that these assumptions may be wrong $\left(\chi_{\text {red }}^{2} \approx 1.2\right)$. We used the LITpro ${ }^{1}$ software (Tallon-Bosc et al. 2008) to extract the best-fit binary

\footnotetext{
1 http://www.jmmc.fr/litpro
}

Table 2. Interferometric best-fit measurements and $1 \sigma$ error-bars.

\begin{tabular}{lcrr}
\hline \hline & & \multicolumn{2}{c}{ Observing date } \\
Parameter & Unit & $2012-06-10$ & \multicolumn{1}{c}{$2012-08-15$} \\
\hline HJD & $(-2450000)$ & 6088.595 & 6154.573 \\
$\left(f_{3} / f_{1+2}\right)_{1.65 \mu}$ & & $0.24 \pm 0.02$ & $0.24 \pm 0.02$ \\
$\delta \mathrm{E}$ & $(\mathrm{mas})$ & $-7.96 \pm 0.16$ & $-6.98 \pm 0.14$ \\
$\delta \mathrm{N}$ & $(\mathrm{mas})$ & $-4.73 \pm 0.09$ & $-5.13 \pm 0.10$ \\
$r$ & $(\mathrm{mas})$ & $9.26 \pm 0.19$ & $8.66 \pm 0.17$ \\
$\theta$ & $\left({ }^{\circ}\right)$ & $239.2 \pm 1.0$ & $233.7 \pm 1.1$ \\
$\chi_{\text {red }}^{2}$ & & 1.2 & 1.4 \\
\hline
\end{tabular}

Table 3. Best-fit circular orbital solutions of the inner pair for the 2011 and 2012 campaigns.

\begin{tabular}{lccc}
\hline \hline Parameter & Unit & Primary & Secondary \\
\hline \multicolumn{4}{c}{ 2011 SB2 solution } \\
$P_{\text {in }}$ & $(\mathrm{d})$ & 2.67454 (fixed) \\
$T_{0}$ & $(\mathrm{HJD}-2450000)$ & $1327.136 \pm 0.006$ \\
$M_{2} / M_{1}$ & \multicolumn{2}{c}{$0.623 \pm 0.008$} \\
$\gamma$ & $\left(\mathrm{km} \mathrm{s}^{-1}\right)$ & $-15.7 \pm 2.4$ & $-12.1 \pm 3.2$ \\
$K$ & $\left(\mathrm{~km} \mathrm{~s}^{-1}\right)$ & $208.3 \pm 2.3$ & $334.5 \pm 3.6$ \\
$\mathrm{rms}$ & $\left(\mathrm{km} \mathrm{s}^{-1}\right)$ & \multicolumn{2}{c}{6.2} \\
\multicolumn{4}{c}{} \\
\multicolumn{4}{c}{$2012 \mathrm{SB} 2$ solution } \\
$P_{\text {in }}$ & $(\mathrm{d})$ & $2.67454($ fixed) \\
$T_{0}$ & $(\mathrm{HJD}-2450000)$ & $1327.151 \pm 0.005$ \\
$M_{2} / M_{1}$ & \multicolumn{2}{c}{$0.630 \pm 0.007$} \\
$\gamma$ & $\left(\mathrm{km} \mathrm{s}^{-1}\right)$ & $-20.4 \pm 2.0$ & $-12.1 \pm 2.8$ \\
$K$ & $\left(\mathrm{~km} \mathrm{~s}^{-1}\right)$ & $214.4 \pm 2.5$ & $340.4 \pm 3.9$ \\
rms & $\left(\mathrm{km} \mathrm{s}^{-1}\right)$ & \multicolumn{2}{c}{13.1} \\
\hline
\end{tabular}

parameters, namely the flux ratio (assumed to be constant across the $H$-band) and the astrometric separation. The closure phases were also analysed independently using the method presented in Absil et al. (2011), and the results agreed excellently.

HD 150136 was clearly resolved on both dates with a slight decrease in separation between June and August (Table 2). Figure 1 shows the data obtained in August 2012 overlaid with the best-fit binary model. The final accuracy is dominated by a $2 \%$ uncertainty in the wavelength calibration of PIONIER. Adopting a distance of $1.32 \pm 0.12 \mathrm{kpc}$ (Herbst \& Havlen 1977), the measured angular separations translate into projected distances of $12.2 \pm 1.1$ and $11.4 \pm 1.1 \mathrm{AU}$ for the June and August observations. The $1 \sigma$ error-bars are dominated by the $10 \%$ uncertainty on the distance.

\section{Orbital properties}

\subsection{The close pair}

We used the Liège Orbital Solution Package ${ }^{2}$ (LOSP) to compute the orbital solutions of the inner pair during the 2011 and 2012 campaigns (Table 3). This provided a measurement of the systemic velocity of the inner pair at these epochs. We fixed the orbital period to the same value as that of Paper I, i.e. 2.67454 days. For both campaigns, the mass ratio, the

2 LOSP is developed and maintained by $\mathrm{H}$. Sana and is available at http://www. science.uva.nl/ hsana/losp.html. The algorithm is based on the generalization of the SB1 method of Wolfe et al. (1967) to the SB2 case along the lines described in Rauw et al. (2000) and Sana et al. (2006). 

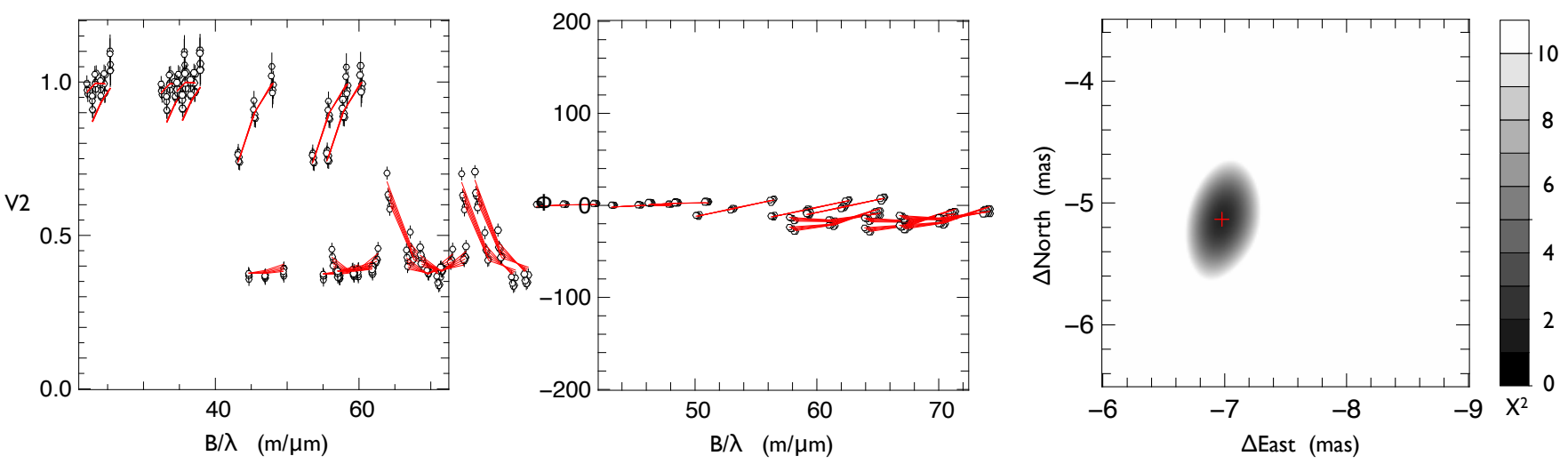

Fig. 1. Calibrated visibilities (left panel) and closure phases (middle panel) from PIONIER in August 2012, overlaid with the best fit binary model (red solid lines). The right panel shows the $\chi^{2}$ map in the vicinity of the best-fit solution.

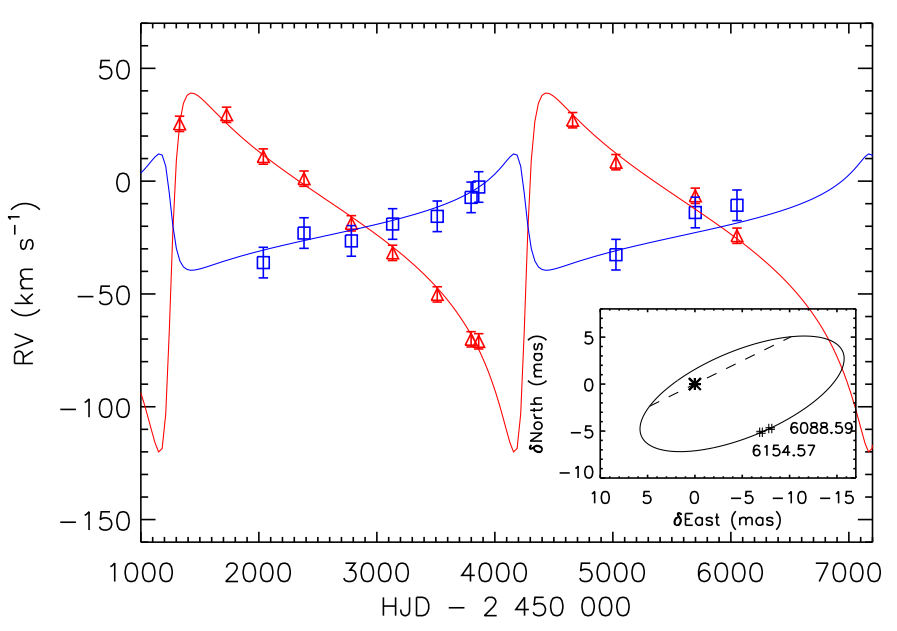

Fig. 2. Main panel: evolution of the third component RVs (triangles) and of the systemic velocity of the $\mathrm{O}+\mathrm{O}$ inner system (squares) as a function of time. The best-fit RV curves are overlaid (Table 5). Insert: PIONIER astrometric points and projection of the best-fit relative orbit on the plane of the sky. The dashed line shows the line of nodes.

semi-amplitudes, and the projected semi-major axis are similar to those found in Paper I. The difference between the systemic velocities of the primary and of the secondary components is larger for the 2012 solution but, globally, both orbital solutions of Table 3 agree with each other and with the solution determined in Paper I.

\subsection{The third companion}

\subsubsection{Spectroscopic orbital solution}

We determined the systemic velocities of the inner system during the individual short-term campaigns with more than one spectrum since 1999. These values are put in perspective with the RVs of the tertiary component in Fig. 2. There is a clear, anti-correlated periodic motion of the systemic velocity of the close binary system and of the tertiary component. We performed a Fourier analysis on the tertiary RVs using the Heck-Manfroid-Mersch method (Heck et al. 1985, refined by Gosset et al. 2001). The highest peak in the periodogram indicates a period of about $3335 \pm 260$ days. We adopted this value as our initial guess and used LOSP to refine the period estimate. We computed an SB1 orbital solution from the RVs of the tertiary alone and an SB2 solution using the tertiary RVs
Table 4. Best-fit LOSP RV solution of the wide system.

\begin{tabular}{lccc}
\hline & & \multicolumn{2}{c}{ LOSP RV solution } \\
Parameter & Unit & Inner pair & Tertiary \\
\hline$P_{\text {out }}$ & $(\mathrm{d})$ & \multicolumn{2}{c}{$2980 \pm 71$} \\
$e$ & & $0.60 \pm 0.14$ \\
$\omega$ & $\left({ }^{\circ}\right)$ & $259.2 \pm 5.8$ \\
$T$ & $(\mathrm{HJD}-2450000)$ & \multicolumn{2}{c}{$1193 \pm 104$} \\
$M_{3} / M_{1+2}$ & & $0.31 \pm 0.07$ \\
$\gamma$ & $\left(\mathrm{km} \mathrm{s}^{-1}\right)$ & $-21.6 \pm 2.7$ & $-16.1 \pm 4.9$ \\
$K$ & $\left(\mathrm{~km} \mathrm{~s}^{-1}\right)$ & $16.7 \pm 4.5$ & $53.6 \pm 14.4$ \\
$\mathrm{rms}$ & $\left(\mathrm{km} \mathrm{s}^{-1}\right)$ & \multicolumn{2}{c}{5.2} \\
\hline
\end{tabular}

and the systemic velocities of the inner pair $\left(\gamma_{12}\right)$. The two solutions are in excellent agreement and the SB2 RV solution is given in Table 4 . The reliability of the RV-only solution, especially its eccentricity and the semi-amplitudes of the RV curves, is unfortunately limited by a lack of sampling around periastron.

\subsubsection{Simultaneous RV and astrometric orbital solution}

The separations measured by PIONIER in June and August 2012 are relatively small and indicate an eccentricity at the upper end of the confidence interval of the RV-only solution of Table 4. We therefore proceeded to simultaneously adjust the RV and astrometric measurements of the outer pair. We minimized the squared differences between the measurements and the model

$$
\begin{aligned}
\chi^{2}= & \sum\left(\frac{\gamma_{12}-\gamma_{12}^{\mathrm{mod}}}{\sigma_{12}}\right)^{2}+\sum\left(\frac{v_{3}-v_{3}^{\mathrm{mod}}}{\sigma_{3}}\right)^{2} \\
& +\sum\left(\frac{\delta E-\delta E^{\mathrm{mod}}}{\sigma_{\delta E}}\right)^{2}+\sum\left(\frac{\delta N-\delta N^{\mathrm{mod}}}{\sigma_{\delta N}}\right)^{2}
\end{aligned}
$$

using a Levenberg-Marquardt method, adopting the RV-only solution of Table 4 as a starting point. Based on the residuals of the tertiary and the systemic RVs around the best-fit RV curves, we adopted $\sigma_{3}=3.4$ and $\sigma_{12}=6.8 \mathrm{~km} \mathrm{~s}^{-1}$ as typical uncertainties on $v_{3}$ and $\gamma_{12}$. The three-dimensional orbital solution converges towards a higher eccentricity (although still within error-bars), hence towards larger RV curve semi-amplitudes, than the RVonly solution. All other parameters remain unchanged. The bestfit combined solution is given in Table 5 and is shown in Fig. 2.

To estimate the uncertainties, we performed Monte-Carlo (MC) simulations. We randomly drew input RVs and astrometric positions around the best-fit orbital solution at epochs 
Table 5. Best-fit simultaneous RV and astrometric orbital solution of the wide system.

\begin{tabular}{lccc}
\hline & & \multicolumn{2}{c}{ Combined solution } \\
Parameter & Unit & Inner pair & Tertiary \\
\hline$P_{\text {out }}$ & $(\mathrm{d})$ & $3008 \pm 54$ \\
$e$ & & $0.73 \pm 0.05$ \\
$\omega$ & $\left(^{\circ}\right)$ & $250.7 \pm 2.9$ \\
$T$ & $(\mathrm{HJD}-2450000)$ & $1241 \pm 38$ \\
$M_{3} / M_{1+2}$ & & $0.32 \pm 0.08$ \\
$i_{\text {out }}$ & $\left({ }^{\circ}\right)$ & $107.9 \pm 3.0$ \\
$\Omega$ & $\left({ }^{\circ}\right)$ & $-60 \pm 10$ \\
& & & \\
$\gamma$ & $\left(\mathrm{km} \mathrm{s}^{-1}\right)$ & $-19.9 \pm 2.2$ & $-21.5 \pm 2.0$ \\
$K$ & $\left(\mathrm{~km} \mathrm{~s}^{-1}\right)$ & $25.8 \pm 7.6$ & $79.8 \pm 11$ \\
$a$ & $\left(\mathrm{~A} . \mathrm{U}^{-}\right)$ & $5.1 \pm 1.2$ & $15.8 \pm 0.6$ \\
$M$ & $\left(M_{\odot}\right)$ & $102 \pm 16$ & $33 \pm 12$ \\
& & \multicolumn{2}{c}{0.94} \\
$\chi_{\text {red }}^{2}$ & & \multicolumn{2}{c}{} \\
\hline
\end{tabular}

Notes. The corresponding RV curves and projected orbit on the plane of sky are displayed in Fig. 2.

corresponding to our observing dates. We used normal distributions with standard deviations corresponding to the $1 \sigma$ measurement uncertainties. We also included the uncertainty on the distance by drawing the distance from a normal distribution centered on $1320 \mathrm{pc}$ and with a standard deviation of $120 \mathrm{pc}$. We recomputed the best-fit orbital solution and inclination using 1000 simulated data sets and we constructed the distributions of the output parameters. The medians of the distributions match the best-fit values of Table 5 very well. We used the distances between the median and the 0.16 and 0.84 percentiles as uncertainty estimates. The upper and lower error-bars agree within $10 \%$ for all parameters; Table 5 provides the average of both values.

\section{Discussion}

\subsection{Physical properties}

Using the best-fit three-dimensional orbit, absolute mass estimates are $M_{3}=33 \pm 12 M_{\odot}$ and $M_{1+2}=102 \pm 16 M_{\odot}$ for the third companion and the total mass of the inner pair, respectively. Combining the total absolute mass of the inner pair with the spectroscopic minimum masses of Paper I (Table 4), we constrain the inclination of the inner binary to values of $49.6 \pm 3.6^{\circ}$. This value is compatible with the absence of eclipse in the inner system. Best masses for the primary and secondary are thus $M_{1}=62.6 \pm 10.0$ and $M_{2}=39.5 \pm 6.3 M_{\odot}$. The agreement with the expected masses given the estimated spectral types is remarkably good (Martins et al. 2005). The dynamical masses also agree within errors with the evolutionary masses obtained in Paper I: $70.6_{-9.1}^{+11.4}, 36.2_{-1.6}^{+5.0}$ and $27.0_{-3.5}^{+3.0} M_{\odot}$ for components 1 , 2 , and 3, respectively. The measured flux-ratio in the $H$-band, $\left(f_{3} / f_{1+2}\right)_{1.65 \mu}=0.24$, also agrees well with the expected $H$-band flux-ratio of 0.26 given the component spectral types (Martins \& Plez 2006), which confirms the main-sequence nature of the tertiary object (see discussion in Paper I).

\subsection{Non-thermal emission}

The present study allows us to clarify to some extent the considerations on the origin of the non-thermal radio emission.
As argued in Paper I, the synchrotron radio emission probably comes from the colliding winds in the wide orbit. The radius of the photosphere $(\tau=1)$ for the primary (whose wind dominates the free-free opacity in the system) is expected to be at most $850 R_{\odot}($ at $\lambda=20 \mathrm{~cm})$, strongly suggesting that the stagnation point of the collision zone must be located farther away (see Paper I). For the tertiary star, we assume two typical values for the mass loss rate: $\dot{M}_{\mathrm{cl}}=10^{-7} M_{\odot} \mathrm{yr}^{-1}$ (classical value, Muijres et al. 2012) and $\dot{M}_{\mathrm{ww}}=10^{-9} M_{\odot} \mathrm{yr}^{-1}$ (more representative of the weak-wind case). We also adopt a terminal velocity of $2500 \mathrm{~km} \mathrm{~s}^{-1}$, corresponding to $2.6 \times v_{\mathrm{esc}}$ and $v_{\mathrm{esc}} \approx 970 \mathrm{~km} \mathrm{~s}^{-1}$ for O6.5-7 V stars (Muijres et al. 2012).

On these bases, we estimate that, in the classical-wind case, the stagnation point is located at about 260 and $1630 R_{\odot}$ from the tertiary at periastron and at apastron. In the weak-wind case, these distances reduce to about 30 and $200 R_{\odot}$. Using the same approach as described in Paper I, we find that the extension of the radio photosphere of the tertiary star is shorter than the distance between the tertiary component and the stagnation point of the colliding winds at shorter wavelengths $(3.6$ and $6 \mathrm{~cm})$, whatever the assumption about the nature of the stellar wind (classical or weak).

In the classical-wind case, however, the stagnation point very close to periastron could be located below the radio photosphere at longer wavelengths (13 and $20 \mathrm{~cm}$ ) where HD 150136 is significantly detected, but the observations reported by Benaglia et al. (2006) were performed in December 2003, which is far from periastron passage according to our ephemeris. In the weak-wind case, the stagnation point is always located significantly outside the photosphere of the dense primary wind, even at periastron, and at all wavelengths. These facts are in agreement with the rather high flux densities measured between 3 and $20 \mathrm{~cm}$, as reported by Benaglia et al. (2006).

As discussed in De Becker et al. (2012) for a similar system, the variability in the synchrotron radio flux from a collidingwind binary most likely comes from two changing factors: stellar separation and free-free absorption. Following the discussion above, absorption is unlikely to dominate. Any temporal change of the HD 150136 flux densities would therefore be mainly attributed to the varying stellar separation along the eccentric wide orbit. One may therefore expect the radio flux density to reach its maximum very close to periastron, with a minimum at apastron.

A radio monitoring of the wide orbit in HD 150136 (so far non-existent) is required to validate this scenario and to achieve a more detailed description of the relevant non-thermal processes. Given the expected location of the stagnation point, the wind interaction zone may be resolvable from the stars by very large baseline radio interferometric facilities. Imaging the wind-wind collision at several epochs would help to distinguish between a weak-wind, as possibly suggested by the CMFGEN analysis in Paper I, and a normal wind for the tertiary star.

\section{Summary}

We reported the very first interferometric detection of the outer companion in the hierarchical triple system HD 150136. Combining the interferometric measurements, new and archival spectroscopy with data from Paper I, we obtained the first threedimensional orbital solution of the wider system. The best-fit solution indicates a 8.2-yr period and a high eccentricity $(e \gtrsim 0.7)$. The accuracy of the PIONIER interferometric measurements allowed us to constrain the inclination of the outer orbit, and from there on, of the inner pair to within a few degrees only. 
We constrained the masses of the three stars of the system to 63,40 , and $33 M_{\odot}$ for the O3-3.5 V, O5.5-6 V, and O6.5-7 V components. In particular, this is the first direct measurement of the mass of an early main-sequence $\mathrm{O}$ star. We showed that the obtained dynamical masses agree within errors with the evolutionary masses estimated in Paper I.

Although relative error-bars remain at the $15 \%$-level for masses of the inner pair components and at the 30\%-level for the tertiary mass, spectroscopic observations around the next periastron passage in 2015 and further interferometric monitoring over a significant fraction of the orbit may provide direct mass measurements with uncertainties of a couple of per cent only. It will also allow for an accurate independent measurement of the distance to the system. This constitutes a must-do to accurately test high-mass star evolutionary models and to investigate the reality and origin of the mass discrepancy in more details (e.g. Herrero et al. 1992; Weidner \& Vink 2010).

As for other non-thermal emitters (HD 93250, Sana et al. 2011; HD 167971, De Becker et al. 2012), the present study also shows that long baseline interferometry is ideally suited to resolve the components of O-type non-thermal radio emitters. The significant advance on the three-dimensional description of the orbits in HD 150136 is a solid base for future investigations to understand the non-thermal emission and particle acceleration processes at work in massive multiple systems.

Acknowledgements. PIONIER is funded by the Université Joseph Fourier (UJF, Grenoble) through its Poles TUNES and SMING and the vice-president of research, the Institut de Planétologie et d'Astrophysique de Grenoble, the "Agence Nationale pour la Recherche" with the program ANR EXOZODI, and the Institut National des Sciences de l'Univers (INSU) with the programs "Programme National de Physique Stellaire" and "Programme National de Planétologie". The integrated optics beam combiner is the result of a collaboration between IPAG and CEA-LETI based on CNES R\&T funding. The authors thank all the people involved in the VLTI project. The use of Yassine Damerdji's orbital code is also warmly acknowledged. This study made use of the Smithsonian/NASA Astrophysics Data System (ADS), of the Centre de Données astronomiques de Strasbourg (CDS) and of the Jean-Marie Mariotti Center (JMMC). Some calculations and graphics were performed with the freeware Yorick.

\section{References}

Absil, O., Le Bouquin, J.-B., Berger, J.-P., et al. 2011, A\&A, 535, A68

Benaglia, P., Koribalski, B., \& Albacete Colombo, J. F. 2006, PASA, 23, 50

De Becker, M. 2007, A\&ARv, 14, 171

De Becker, M., Sana, H., Absil, O., Le Bouquin, J.-B., \& Blomme, R. 2012, MNRAS, 423, 2711

Gosset, E., Royer, P., Rauw, G., Manfroid, J., \& Vreux, J.-M. 2001, MNRAS, 327,435

Haguenauer, P., Alonso, J., Bourget, P., et al. 2010, in SPIE Conf. Ser., 7734, 3

Heck, A., Manfroid, J., \& Mersch, G. 1985, A\&AS, 59, 63

Herbst, W., \& Havlen, R. J. 1977, A\&AS, 30, 279

Herrero, A., Kudritzki, R. P., Vilchez, J. M., et al. 1992, A\&A, 261, 209

Lafrasse, S., Mella, G., Bonneau, D., et al. 2010, in SPIE Conf. Ser., 7734, $44 \mathrm{E}$

Le Bouquin, J.-B., Berger, J.-P., Lazareff, B., et al. 2011, A\&A, 535, A67

Mahy, L., Gosset, E., Sana, H., et al. 2012, A\&A, 540, A97

Martins, F., \& Plez, B. 2006, A\&A, 457, 637

Martins, F., Schaerer, D., \& Hillier, D. 2005, A\&A, 436, 1049

Muijres, L. E., Vink, J. S., de Koter, A., Müller, P. E., \& Langer, N. 2012, A\&A, 537, A37

Rauw, G., Sana, H., Gosset, E., et al. 2000, A\&A, 360, 1003

Sana, H., Gosset, E., \& Rauw, G. 2006, MNRAS, 371, 67

Sana, H., Le Bouquin, J.-B., De Becker, M., et al. 2011, ApJ, 740, L43

Tallon-Bosc, I., Tallon, M., Thiébaut, E., et al. 2008, in SPIE Conf. Ser., 7013, $1 \mathrm{~J}$

Weidner, C., \& Vink, J. S. 2010, A\&A, 524, A98

Wolfe, Jr., R. H., Horak, H. G., \& Storer, N. W. 1967, in Modern Astrophysics. A memorial to Otto Struve, ed. M. Hack (New York: Gordon \& Breach), 251 